Nervenarzt 2018 $\cdot 89: 632-638$ https://doi.org/10.1007/s00115-018-0512-6 Online publiziert: 4. April 2018

(c) Der/die Autor(en) 2018.

CrossMark

A. Tschugg ${ }^{1} \cdot$ B. Meyer ${ }^{2} \cdot$ M. Stoffel ${ }^{3} \cdot$ P. Vajkoczy ${ }^{4} \cdot$ F. Ringel ${ }^{5} \cdot$ S.-O. Eicker ${ }^{6}$.

V. Rhode ${ }^{7} \cdot$ C. Thomé

${ }^{1}$ Universitätsklinik für Neurochirurgie, Medizinische Universität Innsbruck, Innsbruck, Österreich

${ }^{2}$ Neurochirurgische Klinik und Poliklinik, Klinikum rechts der Isar, TU München, München, Deutschland

${ }^{3}$ Klinik für Neurochirurgie, Helios Klinikum Krefeld, Krefeld, Deutschland

${ }^{4}$ Neurochirurgische Klinik, Charité Universitätsmedizin Berlin, Berlin, Deutschland

${ }^{5}$ Neurochirurgische Klinik und Poliklinik, Universitätsmedizin Mainz, Mainz, Deutschland

${ }^{6}$ Klinik für Neurochirurgie, Universitätsklinikum Hamburg-Eppendorf, Hamburg, Deutschland

${ }^{7}$ Klinik für Neurochirurgie, Universitätsmedizin Göttingen, Göttingen, Deutschland

\title{
Operative Versorgung der degenerativen Halswirbelsäule
}

porektomie sowie als dorsale Verfahren die Laminektomie mit Instrumentation oder die Laminoplastie infrage. Die operativen Eingriffe für zervikale degenerative Bandscheibenerkrankungen stiegen beispielsweise in den USA um $67 \%$ in 10 Jahren an [1]. Die anteriore Diskektomie nach Cloward [2] mit Fusion oder die zervikale Arthroplastie werden bei zervikalen Bandscheibenvorfällen häufiger durchgeführt als der dorsale Operationszugang nach Frykholm [3]. Die operative Therapie rein knöcherner zervikaler Foramenstenosen ist Gegenstand kontroverser Diskussionen, zudem finden sich in der Literatur nur wenige evidenzbasierte Daten, welche Operationsmethode die geeignetere darstellt [4]. In der Behandlung der zervikalen Myelopathie werden sowohl die Operationsindikation als auch die verschiedenen ventralen und dorsalen Verfahren mit ihren Vorund Nachteilen nach wie vor diskutiert.

Die Altersstruktur unserer Gesellschaft, die verbesserte perioperative medizinische Versorgung sowie auch die erhöhte Erwartungshaltung der Patienten an eine verbesserte Lebensqualität verlangen neben konservativen Therapieoptionen immer häufiger eine operative Intervention. Die anteriore zervikale Diskektomie mit Fusion (ACDF), die zervikale Arthroplastie und die posteriore zervikale Foraminotomie stellen die möglichen operativen Optionen für eine zervikale Radikulopathie dar. Bei zervikaler Myelopathie kommen neben der ACDF, die Kor- den. Hier sind regelmäßige Kontrolluntersuchungen zu empfehlen [5].

Bei einer Zervikobrachialgie ohne motorisches Defizit wird primär ein konservatives Therapieregime empfohlen [6]:

- frühzeitige schonende Mobilisation,

- Schmerztherapie nach dem Schema der World Health Organization (WHO),

- physikalische Maßnahmen: Wärmeapplikation, leichte Massagen, Rückenschule,

- Myotonolytika bei paravertebralem Hartspann.

\section{Indikationsstellung zur operativen Versorgung}

Eine chirurgische Therapie der zervikalen Radikulopathie ist bei therapieresistenten Schmerzen oder begleitender progressiver klinischer Symptomatik mit sensomotorischem Defizit indiziert. Der Behandlungsalgorithmus einer Zervikobrachialgie ist in 0 Abb. 1 beschrieben. Da die Radikulopathie durch einen Bandscheibenvorfall ähnlich wie der lumbale Bandscheibenvorfall häufig gut auf eine nichtoperative Therapie anspricht, wird in der Regel eine intensive konservative Therapie ggf. mit periradikulärer Infiltration für mindestens 6 bis 12 Wochen durchgeführt. Bei relevantem motorischem Defizit wird eine dringliche Operationsindikation gestellt. 


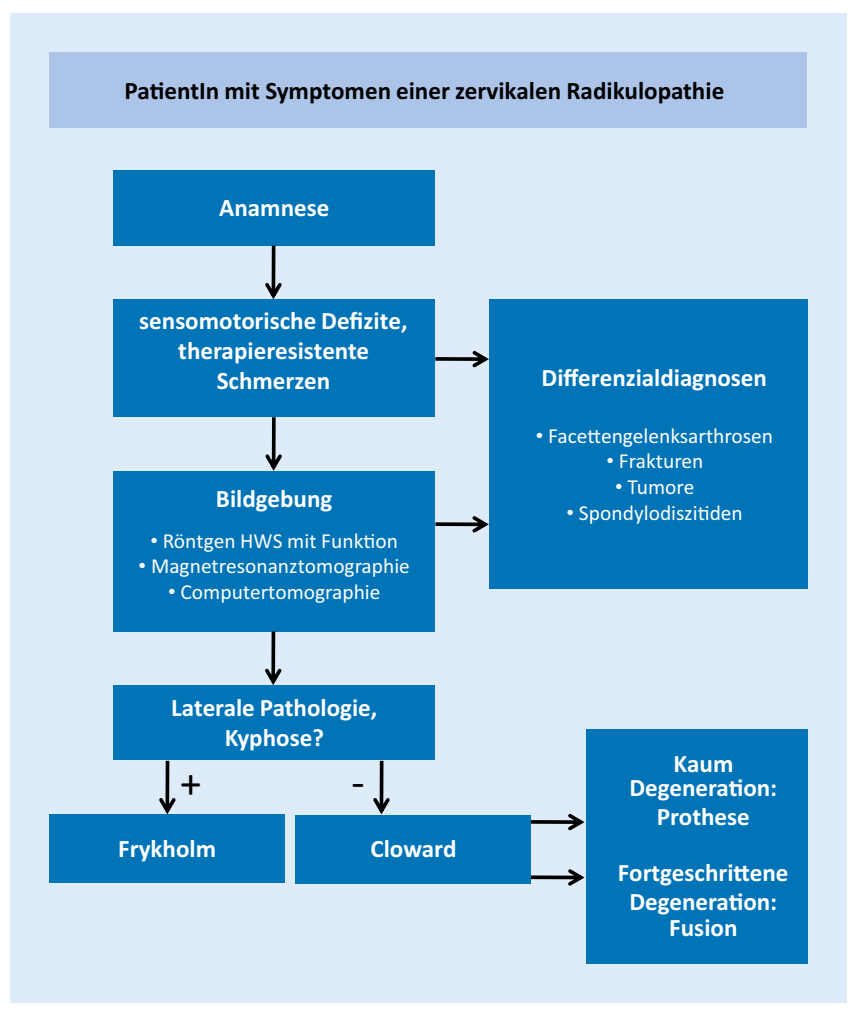

Abb. $1 \triangleleft$ Behandlungsalgorithmus bei zervikaler Radikulopathie. HWS Halswirbelsäule
Der natürliche Verlauf der zervikalen Myelopathie wird nach wie vor kontrovers beurteilt. Im Falle einer moderaten oder ausgeprägten Symptomatik ist die progrediente klinische Verschlechterung inzwischen unstrittig [7], sodass eine klare Operationsindikation besteht. Bei milder zervikaler Myelopathie sind variable Verläufe beschrieben und manche Patienten zeigen einen stabilen Verlauf [8], der zu abwartender Haltung verleitet. Leider kommt es in $75 \%$ der Fälle $\mathrm{zu}$ einer episodischen Verschlechterung der zervikalen Myelopathie, die sich nach einer Dekompression häufig nur unzureichend erholt [9]. Vor diesem Hintergrund wird heutzutage eine chirurgische Dekompression bei eindeutiger klinischer Myelopathie auch geringer Ausprägung (e.g. Feinmotorikstörungen) empfohlen. Eine prophylaktische Operationsindikation bei asymptomatischen Patienten besteht hingegen auch bei eindeutiger Spinalkanalstenose nicht, zumal nur ein geringer Prozentsatz dieser Patienten innerhalb eines Jahres Symptome entwickeln wird [10]. Als Hilfsmittel zur Identifikation dieser Patienten haben sich die evozierten Potenziale herauskristallisiert. Bei pathologischen somatosen- sorisch evozierten Potenzialen (SSEPs) trotz fehlender Klinik muss von einer höheren Progressionsrate ausgegangen werden [11]. Als weitere Risikofaktoren für eine klinische Verschlechterung wurden eine erhöhte Segmentmobilität, eine segmentale Kyphose oder Olisthese sowie eine trianguläre Verformung des Myelons identifiziert [12]. Damit hat sich die Datenlage zum Management bei milder myelopathischer Symptomatik zunehmend zur frühzeitigen, aber nicht prophylaktischen chirurgischen Dekompression verschoben.

\section{Präoperative Planung und Diagnosestellung}

Neben der Anamnese und klinisch-neurologischen Untersuchung der Patienten sind bildgebende Verfahren zur weiteren Diagnosestellung unerlässlich. Native Röntgenaufnahmen in anterior-posteriorer, seitlicher und Extensions-Flexions-Stellung erlauben die Beurteilung der knöchernen Strukturen, des sagittalen Profils sowie potenzieller Instabilitäten. Die Magnetresonanztomographie in T1- und T2-gewichteten Sequenzen eignet sich zur Darstellung der Weichteil- strukturen, insbesondere zur Diagnostik von Bandscheibenvorfällen und von Veränderungen des Myelons bei zervikaler Myelopathie. Die Computertomographie stellt zur Beurteilung ossärer degenerativer Veränderungen, insbesondere osteophytärer Anbauten sowie knöcherner Neuroforamenstenosen, eine weitere wichtige Diagnostik zur präoperativen Planung dar (• Abb. 2).

\section{Operatives Verfahren bei zervikaler Radikulopathie}

Die ventrale Diskektomie und Fusion (ACDF) erfolgt in Rückenlage mit paramedianer Hautinzision und Eröffnen von Subkutis und Platysma. Nach stumpfer Präparation bis zum entsprechenden Bandscheibenfach wird dieses mit verschiedenen Instrumenten ausgeräumt. Osteophytäre Anbauten werden mittels Diamantfräse abgetragen bis das hintere Längsband zum Vorschein kommt und in der Regel entfernt wird, sodass die Dekompression aller nervalen Strukturen sichergestellt ist. Nach Reinigung der Grund- und Deckplatte mittels Kürette erfolgt das Einbringen eines Cages oder einer Bandscheibenendoprothese unter Bildwandlerkontrolle [2]. Der Operationszugang ist in - Abb. 3 dargestellt.

Bei der dorsalen Foraminotomie nach Frykholm wird zwischen dem traditionellen medianen Zugang und dem minimal-invasiven transtubulären, paramedianen Zugang unterschieden. Letzterer wird in der Literatur zunehmend propagiert. In Bauchlage oder in sitzender Position erfolgt die Schnittführung $2 \mathrm{~cm}$ paramedian über dem zu operierenden Segment, sodass unter Bildwandlerkontrolle das Tubussystem in Dilatationstechnik eingebracht werden kann. Mittels Diamantfräse und unter Zuhilfenahme des Mikroskops wird eine Fensterung am Übergang der Wirbelbögen zu den Gelenkflächen durchgeführt und der Rand des Duralsacks mit der abgehenden Nervenwurzel aufgesucht. Bei weichen Bandscheibenvorfällen wird der Sequester mobilisiert und entfernt, im Falle einer knöchernen Neuroforamenstenose eine dorsale Dekompression des Neuroforamens vorgenommen [3]. Der Operationszugang ist in $\mathbf{A b b} \mathbf{3}$ dargestellt. 


\section{Prothese vs. Fusion}

Grundlage jeder Operation von ventral sollte die Diskektomie, die Foraminotomie sowie die Entfernung des hinteren Längsbandes sein. Eine Fusionsoperation dient der langfristigen Versteifung des operierten Segments durch knöcherne Überbauung der angrenzenden Wirbelkörper mithilfe eines stabilisierenden Interponats. Der historisch verwendete Beckenkammspan wurde durch Cages aus Titan oder Polyetheretherketon (PEEK) weitestgehend ersetzt. Eine zusätzliche Plattenosteosynthese wird in Fällen einer segmentalen Instabilität oder einer multisegmentalen Versorgung empfohlen. Die Ergebnisse zeigen eine sehr gute Patientenzufriedenheit, können jedoch durch Verlust der segmentalen Mobilität im Verlauf zu Anschlussdegenerationen der angrenzenden Segmente führen und damit Reoperationen zur Folge haben [13].

Im Gegensatz zur Fusion kann die Bandscheibenprothese eine Bewegung im entsprechenden Segment gewährleisten [14]. Wesentliche Kontraindikationen für die Implantation einer Prothese sind neben segmentaler Instabilität schwere Facettengelenks- und Bandscheibendegeneration, Deformitäten, Ossifikation des hinteren Längsbandes, traumatische Läsionen der Halswirbelsäule, Osteoporose sowie lokalisierte spinale Infektionen [15].

\section{》) Bei der zervikalen \\ Arthroplastie ist die geeignete \\ Patientenpopulation zu beachten}

Auch der Einsatz bei zervikaler Myelopathie wird von vielen Autoren kritisch beurteilt, auch wenn die US-amerikanischen Zulassungsstudien diese Patienten mit einbezogen haben. Ungeachtet der eingeschränkten Indikationen bleibt festzuhalten, dass wohl für kein wirbelsäulenchirurgisches Verfahren in den letzten Jahren so viel Evidenz generiert wurde wie für die zervikale Arthoplastie, auch wenn ein relevanter Bias nicht auszuschließen ist [16]. In der Zusammenschau aller Studien kann dennoch dem bewegungserhaltenden

Nervenarzt 2018 $\cdot 89: 632-638$ https://doi.org/10.1007/s00115-018-0512-6

(c) Der/die Autor(en) 2018.

\section{A. Tschugg · B. Meyer · M. Stoffel · P. Vajkoczy · F. Ringel · S.-O. Eicker · V. Rhode · C. Thomé Operative Versorgung der degenerativen Halswirbelsäule}

\section{Zusammenfassung}

Hintergrund. Degenerative Veränderungen der Halswirbelsäule umfassen Bandscheibenvorfälle sowie Stenosen des Spinalkanals und/oder der Neuroforamina. Bei lateraler bzw. mediolateraler Kompression einer Nervenwurzel ist eine zervikale Radikulopathie die Folge, die im Falle relevanter motorischer Defizite oder therapierefraktärer Schmerzen eine Operationsindikation nach sich zieht. Bei medianer raumfordernder Wirkung auf das Rückenmark kommt es zur zervikalen Myelopathie, die typischerweise episodisch progredient verläuft und daher bei eindeutigen klinischen Zeichen einer Myelopathie operiert werden sollte.

Ziel der Arbeit. Ziel dieser Arbeit ist es, einen Überblick über die operativen Möglichkeiten, deren Komplikationen und das Outcome für Patienten mit zervikaler Radikulopathie und Myelopathie zu geben.

Material und Methoden. Für das vorliegende Manuskript wurde eine selektive Literaturrecherche in PubMed durchgeführt, die einzelnen Veröffentlichungen für die beste
Evidenz analysiert und mit der Erfahrung der Autoren kombiniert.

Ergebnisse und Diskussion. Die anteriore zervikale Diskektomie mit Fusion (ACDF), die zervikale Arthroplastie und die posteriore zervikale Foraminotomie stellen die bevorzugten operativen Optionen zur Behandlung der Radikulopathie dar. Bei Spinalkanalstenosen kommen neben der ACDF die Korporektomie sowie als dorsale Verfahren die Laminektomie mit Instrumentation oder die Laminoplastie infrage. Bei den klinischen Ergebnissen ist nach Pathologie sowie der Art des operativen Zugangs zu unterscheiden. Die Langzeitverläufe für dorsale und ventrale Operationstechniken an der Halswirbelsäule sind sehr gut, auch wenn bestimmte Konstellationen sich eher für das eine oder das andere Verfahren anbieten.

\section{Schlüsselwörter}

Anteriore Diskektomie und Fusion · Bandscheibenprothese - Zervikale Foraminotomie . Laminektomie - Laminoplastie

\section{Operative treatment of the degenerative cervical spine}

\section{Abstract}

Background. Degenerative alterations of the cervical spine often entail disc herniations and stenoses of the spinal canal and/or neural foramen. Mediolateral or lateral compression of nerve roots causes cervical radiculopathy, which is an indication for surgery in cases of significant motor deficits or refractory pain. Median canal encroachment may result in compression of the spinal cord and cervical myelopathy. Its natural history is typically characterized by episodic deterioration, so that surgical decompression is indicated in cases of clear myelopathic signs.

Objective. The aim of the present article is to outline the operative options for patients with cervical radiculopathy and myelopathy. Furthermore, we describe the operative complications and the outcome in these patients.

Material and methods. For this manuscript a systematic PubMed search was carried out, the papers were systematically analyzed for the best evidence and this was combined with the authors' experience.

Results and conclusion. Depending on the cervical pathology, the most prevalent surgical options for radiculopathy include anterior cervical discectomy and fusion (ACDF), cervical arthroplasty or posterior cervical foraminotomy. Cervical myelopathy may be decompressed by ACDF, corpectomy or posterior approaches like laminectomy plus instrumented fusion or laminoplasty. The outcome depends on the cervical pathology and the type of operation. Overall, in longterm follow-up studies the results of all surgical techniques on the cervical spine are generally considered to be very good, although specific patient characteristics are more suited for a particular approach.

\section{Keywords}

Anterior discectomy and fusion - Intervertebral disk prosthesis . Cervical foraminotomy . Laminectomy · Laminoplasty 

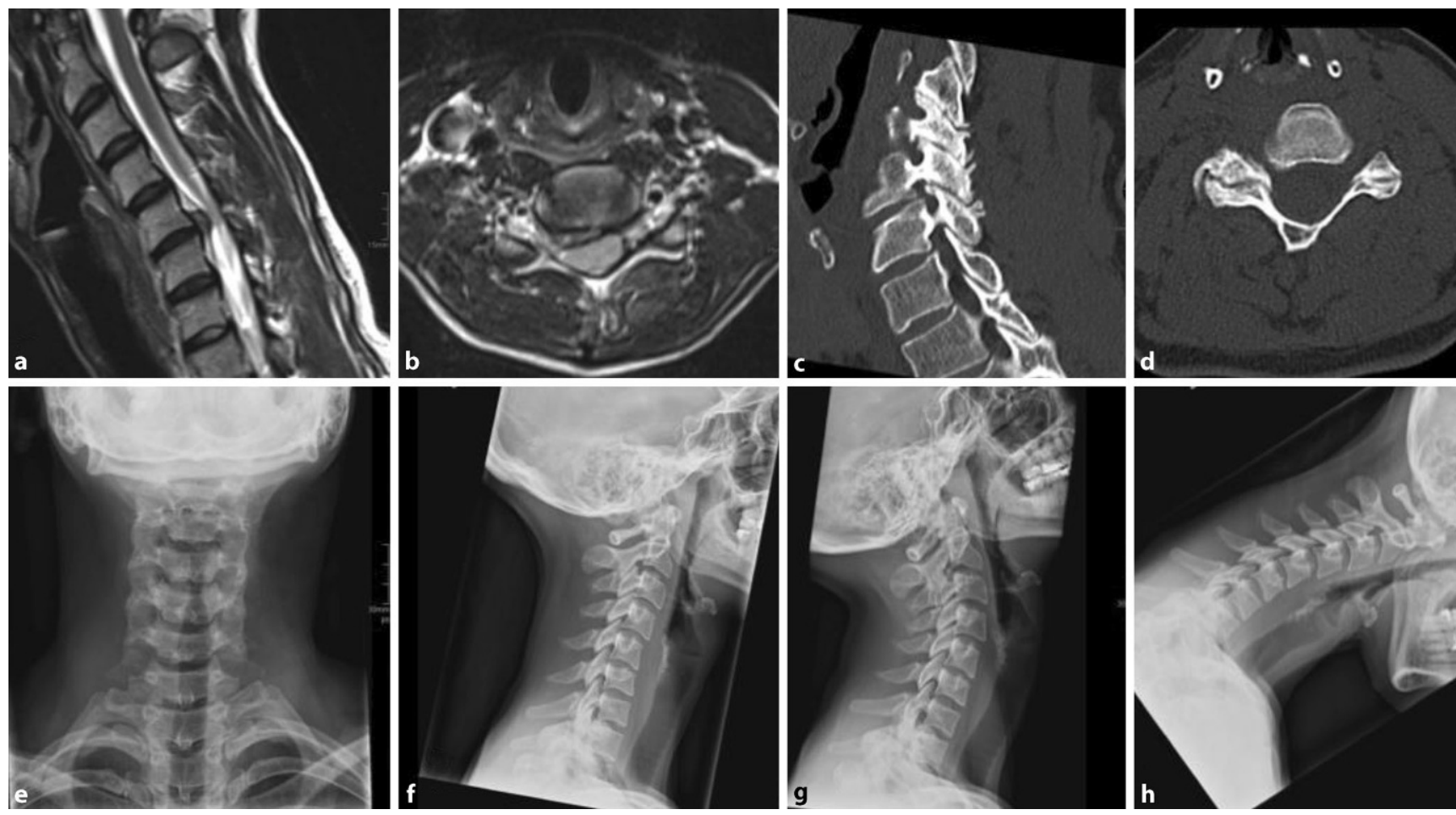

Abb. $2 \Delta$ Zervikaler Bandscheibenvorfall im Magnetresonanztomogramm der Halswirbelsäule in T2-Gewichtung in a sagittaler und $\mathbf{b}$ axialer Schnittebene. Eine knöcherne Neuroforamenstenose kann im Computertomogramm der Halswirbelsäule c sagittal und $\mathbf{d}$ axial dargestellt werden. Ergänzend dazu ein Röntgen der Halswirbelsäule in e anterior-posterioren, $\mathbf{f}$ seitlichen, $\mathbf{g}$ Extensions- und $\mathbf{h}$ Flexionsaufnahmen
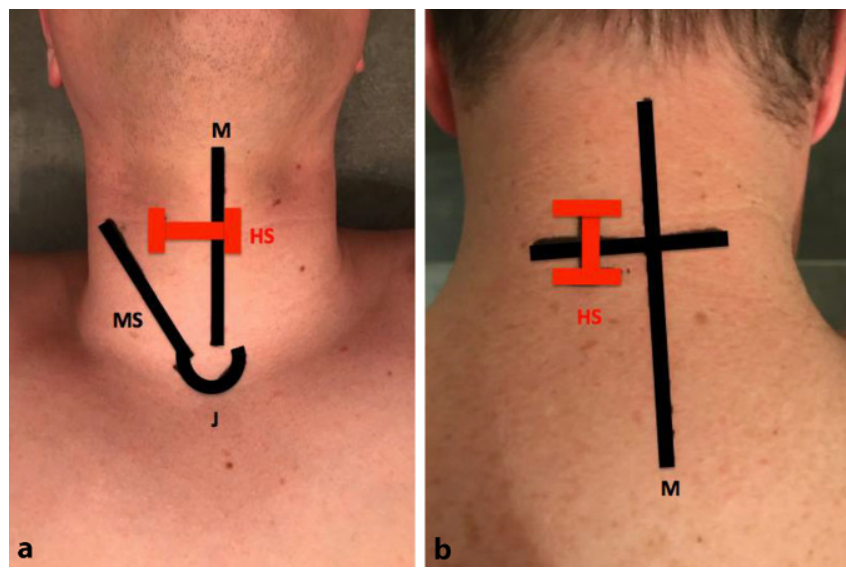

Abb. $3<$ a Zugang nach Cloward, b Zugang nach Frykholm. MS Musculus sternocleidomastoideus, $M$ Mittellinie, HS Hautschnitt, J Jugulum
Verfahren in den Langzeitergebnissen ein signifikanter Vorteil im Vergleich zur anterioren Diskektomie und Fusion bei geeigneten Patienten attestiert werden [17]. Weiterentwicklungen der Bandscheibenprothesen könnten diese Entwicklung weiter forcieren. Allerdings gilt es, die geeignete Patientenpopulation für die zervikale Arthroplastie zu beachten. Jüngere Patienten mit weichen Bandscheibenvorfällen und/oder wenig segmentaler Degeneration eignen sich für ein mobilitätserhaltendes Verfahren, andere eher nicht.

\section{Ventrale Diskektomie vs. Foraminotomie}

In den letzten Jahrzehnten hat sich die ventrale Diskektomie mit Fusion oder Bandscheibenprothese zunehmend verbreitet und gute Ergebnisse erzielt. Die Veränderungen der Biomechanik führen jedoch zum Problem der Anschlussdegeneration und entsprechenden Nachope- rationen. Die dorsale Foraminotomie scheint im Langzeitverlauf eine noch höhere Erfolgsquote aufzuweisen [18], kommt aber wiederum nur für ein selektioniertes Patientengut infrage. Ideal eignet sich das Verfahren für weiche laterale Vorfälle [19]. Deutliche degenerative Veränderungen, starke Nackenschmerzen und ein enger Spinalkanal sprechen gegen die minimal-invasive dorsale Entlastung.

\section{Operative Verfahren bei zervikaler Myelopathie}

Im Falle einer zervikalen Myelopathie finden sich neben einer auf die Bandscheibenfächer begrenzten Kompression des Rückenmarks auch langstreckigere Rückenmarkkompressionen, die von ventral Korporektomien oder von dorsal Laminektomien oder Laminoplastien erforderlich machen. Im Falle einer zervikalen Korporektomie werden über diesen Zugang zwei benachbarte Bandscheibenfächer ausgeräumt und der mediane Anteil des dazwischenliegenden Wirbelkörpers abgetragen und durch ein Wirbel- 
Tab. 1 Gesamtkomplikationsraten für dorsale und ventrale Eingriffe

\begin{tabular}{lll}
\hline Komplikationen & Häufigkeit \\
\hline Komplikationen und Risiken im Rahmen des ventralen Zugangs [21] & $14,5 \%$ \\
\hline Infektionsrate [21] & $1,6 \%$ \\
\hline Heiserkeit (durch eine Schädigung des N. recurrens; [22]) & $3-11 \%$ \\
\hline Dysphagie [23] & Transient: 0-75\%, perma- \\
& nent: 1-7\% \\
\hline C5-Lähmung [24] & $2,5 \%$ \\
\hline Horner-Syndrom [25] & $<1 \%$ \\
\hline Nerven- oder Rückenmarksschädigungen [25] & $<0,5 \%$ \\
\hline Pseudoarthrosis [26] & $2,6-10 \%$ \\
\hline Verletzungen des Ösophagus und der Trachea [25] & $<0,3 \%$ \\
\hline Anschlussdegeneration nach unisegmentaler Fusion (7 Jahre; [27]) & Jährlich: 2,9\%; 10 Jahre: \\
& $25,6 \% ;$ Reoperationsrate: \\
\hline Komplikationen und Risiken im Rahmen des dorsalen Zugangs [21] & $7,5 \%$ \\
\hline Infektionsrate [21] & $13,3 \%$ \\
\hline C5-Lähmung bei operativer Versorgung im Segment C4/5 [28] & $5 \%$ \\
\hline Anschlussinstabilitätsratenach unisegmentaler Dekompression [29] & $0-50 \%$ \\
\hline Jährlich: 0,7\%; 10 Jahre: \\
\hline Persistierende Nacken- und Schulterschmerzen [30] & $3,7 \% ;$ Reoperationsrate: \\
\hline Verletzungen der Arteria vertebralis & $18 \%$ \\
\hline & $<1 \%$ \\
\hline
\end{tabular}

körperersatzimplantat ersetzt. Aus Stabilitätsgründen ist bei Korporektomien zusätzlich eine ventrale Plattenosteosynthese erforderlich.

Eine Laminektomie beinhaltet die Entfernung der Dornfortsätze und der Laminae der entsprechenden Wirbelkörper und wird speziell bei nicht erhaltener zervikaler Lordose mit dem Risiko einer postoperativen Instabilität assoziiert. Dementsprechend kommen eine zusätzliche dorsale Instrumentation mit Massa-lateralis-Schrauben oder minimal-invasive Verfahren wie die „skip laminectomy“ zur Anwendung [20]. Im Rahmen einer Laminoplastie wird mit verschiedene Techniken (z. B. „open door laminoplasty“) der Spinalkanal erweitert, ohne die Wirbelbögen vollständig zu entfernen.

\section{》) Die Auswahl des operativen Verfahrens ist eine individuell zu treffende Entscheidung}

Die Auswahl des operativen Verfahrens (ventral oder dorsal) hängt vom Ursprung der Kompression (von vorne oder von hinten), von der Stellung der
Halswirbelsäule (Kyphose oder Lordose), von der Knochenqualität und damit dem Alter (jung oder alt) sowie von der Anzahl der betroffenen Segmente (monobzw. bisegmental oder multisegmental) $\mathrm{ab}$ und ist damit eine individuell zu treffende Entscheidung. Gemäß Datenlage sollten multisegmentale Laminektomien mit einer dorsalen Instrumentation kombiniert werden.

\section{Komplikationen}

Neben den allgemeinen Operationsrisiken müssen die Komplikationen für den vorderen und hinteren Zugang getrennt voneinander besprochen werden, wobei die Gesamtkomplikationsraten für dorsale und ventrale Eingriffe äquivalent sind ([21]; - Tab. 1).

\section{Prognose}

Beim Outcome ist nach Pathologie sowie der Art des operativen Zugangs zu unterscheiden. Die Langzeitverläufe bei Diskektomie und ventraler Fusion sind insgesamt sehr gut. Entscheidend dabei sind neben der ausreichenden Dekompression der Neuroforamina die Auswahl des Interponats sowie dessen Positionierung und dadurch die Wiederherstellung des sagittalen Profils. Beim Einbringen des Interponats sollte eine Überdistraktion ebenso wie eine Kyphosierung des Segmentes unbedingt vermieden werden. Die wenigen Studien, die anteriore Foraminotomien mit Fusion untersuchten, zeigen ebenfalls ein gutes Outcome in $83-91 \%$ der Fälle mit Revisionsraten von $4-14 \%$ [31]. Die klinischen Langzeitverläufe für die dorsalen Zugänge im Sinne einer posterioren Foraminotomie berichten ebenfalls über ein gutes Outcome in 64-96\% der Patienten und von Revisionsoperationen in 4-7\% der Fälle [32]. Auch die klinischen Verläufe im Rahmen der Bandscheibenendoprothesen sind erfreulich. Durch den Erhalt des Bewegungssegmentes kann die Belastung für die benachbarten Segmente reduziert und insbesondere bei jüngeren Patienten eine Anschlussdegeneration vermieden werden [33].

\section{》) Prognostisch ist die zervikale Myelopathie weniger günstig als die Radikulopathie}

In der Behandlung der zervikalen Myelopathie konnte bislang keine Überlegenheit der ventralen oder der dorsalen Verfahren erhoben werden. In den letzten Jahren haben sich dorsale Techniken wieder mehr verbreitet, da zusätzliche Instrumentationen als Standardverfahren eingesetzt werden und langstreckige ventrale Eingriffe doch eine relevante Komplikationsrate aufweisen. Allerdings handelt es sich um eine sehr heterogene Patientenpopulation, die sich je nach individuellen Charakteristika mehr für ein ventrales oder dorsales Verfahren anbieten, sodass ein direkter Vergleich kaum möglich ist. Prognostisch ist die zervikale Myelopathie weniger günstig als die zervikale Radikulopathie. Obgleich sich die Mehrzahl der Patienten klinisch bessert, bleibt häufig eine myelopathische Restsymptomatik zurück, die gerade bei präoperativ moderater oder schwerer Myelopathie erheblich sein kann. Dieser Umstand hat zur frühzeitigen Indikationsstellung zur Dekompression auch bei milder zervikaler Myelopathie beigetragen. 


\section{Fazit für die Praxis}

\section{- Die Langzeitverläufe bei Diskek-} tomie und ventraler Fusion sind insgesamt sehr gut. Entscheidend sind neben der ausreichenden Dekompression der Neuroforamina die Auswahl des Interponats sowie dessen Positionierung und dadurch die Wiederherstellung des sagittalen Profils. Die klinischen Langzeitverläufe für die dorsalen Zugänge im Sinne einer posterioren Foraminotomie berichten ebenfalls über ein gutes Outcome.

- In der Behandlung der zervikalen Myelopathie konnte bislang keine Überlegenheit der ventralen oder der dorsalen Verfahren erhoben werden. In den letzten Jahren haben sich dorsale Techniken wieder mehr verbreitet, da zusätzliche Instrumentationen als Standardverfahren eingesetzt werden und langstreckige ventrale Eingriffe eine relevante Komplikationsrate aufweisen.

\section{Korrespondenzadresse}

\section{PD Dr. A. Tschugg, Ph.D.}

Universitätsklinik für Neurochirurgie,

Medizinische Universität Innsbruck Anichstr. 35, 6020 Innsbruck, Österreich anja.tschugg@i-med.ac.at

Funding. Open access funding provided by University of Innsbruck and Medical University of Innsbruck.

\section{Einhaltung ethischer Richtlinien}

Interessenkonflikt. A. Tschugg ist Beraterin für Brainlab AG (Feldkirchen, Deutschland). B. Meyer ist Berater für Medtronic (Meerbusch, Deutschland), SpineArt (Frankfurt am Main, Deutschland), Relievant (Sunnyvale, USA). M. Stoffel hat Referentenhonorare von Ulrich Medical (UIm, Deutschland) erhalten. P. Vajkoczy ist Berater für Brainlab (München, Deutschland), Zeiss (Oberkochen, Deutschland), Relievant Medsystems (Redwood City, USA), SpineArt (Plan les Oautes, CH), Ulrich Medical (UIm, Deutschland). S.-O. Eicker ist Berater für Spineart (Plan les Oautes, Schweiz). V. Rhode ist Berater für Ulrich (Ulm, Deutschland), Codman (Norderstedt, Deutschland), BBraun Aesculap (Melsungen, Deutschland). C. Thomé ist Berater für BBraun Aesculap (Melsungen, Deutschland), DepuySynthes (West Chester, USA) und Signus Medizintechnik (Alzenau,
Deutschland). F. Ringel gibt an, dass kein Interessenkonflikt besteht.

Dieser Beitrag beinhaltet keine von den Autoren durchgeführten Studien an Menschen oder Tieren.

Open Access. Dieser Artikel wird unter der Creative Commons Namensnennung 4.0 International Lizenz (http://creativecommons.org/licenses/by/4.0/deed. de) veröffentlicht, welche die Nutzung, Vervielfältigung, Bearbeitung, Verbreitung und Wiedergabe in jeglichem Medium und Format erlaubt, sofern Sie den/die ursprünglichen Autor(en) und die Quelle ordnungsgemäßnennen, einen Linkzur Creative Commons Lizenz beifügen und angeben, ob Änderungen vorgenommen wurden.

\section{Literatur}

1. McGuire KJ, Harrast J, Herkowitz $\mathrm{H}$, Weinstein JN (2012) Geographic variation in the surgical treatment of degenerative cervical disc disease: American Board of Orthopedic Surgery Quality Improvement Initiative; part II candidates. Spine (Phila Pa 1976) 37:57-66

2. Cloward RB (2007) The anterior approach for removal of ruptured cervical disks. 1958. J Neurosurg Spine 6:496-511

3. Frykholm R (1947) Deformities of dural pouches and strictures of dural sheaths in the cervical region producing nerve-root compression; a contribution to the etiology and operative treatment of brachial neuralgia. J Neurosurg 4:403-413

Hier steht eine Anzeige. 
4. Tschugg A, Neururer S, Scheufler KM, Ulmer H, Thomé C, Hegewald AA (2014) Comparison of posterior foraminotomy and anterior foraminotomy with fusion for treating spondylotic foraminal stenosis of the cervical spine: study protocol for a randomized controlled trial (ForaC). Trials 15:437

5. Matz PG (2006) Does nonoperative management play a role in the treatment of cervical spondylotic myelopathy? Spine J6:175S-181S

6. Kjaer $\mathrm{P}$, Kongsted A, Hartvigsen J, IsenbergJørgensen A, Schiøttz-Christensen B, Søborg B et al (2017) National clinical guidelines for non-surgical treatment of patients with recent onset neck pain or cervical radiculopathy. Eur Spine J. https://doi. org/10.1007/s00586-017-5121-8

7. Yarbrough CK, Murphy RK, Ray WZ, Stewart T (2012) The natural history and clinical presentation of cervical spondylotic myelopathy. Adv Orthop. https://doi.org/10.1155/2012/480643

8. Kadanka Z, Mares M, Bednarík J, Smrcka V, Krbec M, Chaloupka R et al (2005) Predictive factors for spondylotic cervical myelopathy treated conservatively or surgically. Eur J Neurol 12:55-63

9. Edwards CC, Riew KD, Anderson PA, Hilibrand AS, Vaccaro AF (2003) Cervical myelopathy. current diagnostic and treatment strategies. Spine J 3:68-81

10. Bednarik J, Kadanka Z, Dusek L, Novotny O, Surelova D, Urbanek I et al (2004) Presymptomatic spondylotic cervical cord compression. Spine (Phila Pa 1976) 29:2260-2269

11. Matz PG, Holly LT, Groff MW, Vresilovic EJ, Anderson PA, Heary RF et al (2009) Joint section on disorders of the spine and peripheral nerves of the American Association of Neurological Surgeons and Congress of Neurological Surgeons: Indications for anterior cervical decompression for the treatment of cervical degenerative radiculopathy. J Neurosurg Spine 11:174-182

12. Oshima Y, Seichi A, Takeshita K, Chikuda H, Ono T, Baba S et al (2012) Natural course and prognostic factors in patients with mild cervical spondylotic myelopathy with increased signal intensity on T2-weighted magnetic resonance imaging. Spine (Phila Pa 1976) 37:1909-1913

13. Sasso RC, Anderson PA, Riew KD, Heller JG (2011) Results of cervical arthroplasty compared with anterior discectomy and fusion: four-year clinical outcomes in a prospective, randomized controlled trial. Orthopedics 34:889

14. Boselie TFM, van Santbrink H (2016) Arthroplasty in cervical degenerative disc disease: fulfilling its long-term promise? J Spine Surg 2(4):359-361

15. Obernauer J, Landscheidt J, Hartmann S, Schubert GA, Thomé C, Lumenta C (2016) Cervical arthroplasty with $\mathrm{ROTAIO}{ }^{\circledR}$ cervical disc prosthesis: first clinical and radiographic outcome analysis in a multicenter prospective trial. BMC Musculoskelet Disord 17:11

16. Bartels RH, Donk R, Verbeek AL (2010) No justification for cervical disk prostheses in clinical practice: a meta-analysis of randomized controlled trials. Neurosurgery 66:1153-1160 (discussion 1160)

17. Gao F, MaoT, Sun W, Guo W, Wang Y, LiZ et al (2015) An updated meta-analysis comparing artificial Cervical Disc Arthroplasty (CDA) versus Anterior Cervical Discectomy and Fusion (ACDF) for the treatment of Cervical Degenerative Disc Disease (CDDD). Spine (Phila Pa 1976) 40:1816-1823

18. Dohrmann GJ, Hsieh JC (2014) Long-term results of anterior versus posterior operations for herniated cervical discs: analysis of 6,000 patients. Med Princ Pract 23:70-73
19. Church EW, Halpern $\mathrm{CH}$, Faught RW, Balmuri U, Attiah MA, Hayden S et al (2014) Cervical laminoforaminotomy for radiculopathy: symptomatic and functional outcomes in a large cohort with longterm follow-up. Surg Neurol Int 5:S536-S543

20. Shiraishi T (2002) Skip laminectomy - a new treatment for cervical spondylotic myelopathy, preserving bilateral muscular attachments to the spinous processes: a preliminary report. Spine J 2:108-115

21. Kato $S$, Nouri A, Wu D, Nori S, Tetreault L, Fehlings MG (2017) Comparison of anterior and posterior surgery for degenerative cervical myelopathy: an MRI-based propensity-score-matched analysis using data from the prospective multicenter AOspine CSM north america and international studies. JBone Joint Surg Am 99:1013

22. Apfelbaum RI, Kriskovich MD, Haller JR (2000) On the incidence, cause, and prevention of recurrent laryngeal nerve palsies during anterior cervical spine surgery. Spine (Phila Pa 1976) 25:2906-2912

23. Joaquim AF, Murar J, Savage JW, Patel AA (2014) Dysphagia after anterior cervical spine surgery: a systematic review of potential preventative measures. Spine J 14:2246-2260

24. Lim CH, Roh SW, Rhim SC, Jeon SR (2017) Clinical analysis of $\mathrm{C} 5$ palsy after cervical decompression surgery: relationship between recovery duration and clinical and radiological factors. Eur Spine J 26:1101-1110

25. Fountas KN, Kapsalaki EZ, Nikolakakos LG, Smisson $\mathrm{HF}$, Johnston KW, Grigorian AA et al (2007) Anterior cervical discectomy and fusion associated complications. Spine (Phila Pa 1976) 32:2310-2317

26. Shriver MF, Lewis DJ, Kshettry VR, Rosenbaum BP, Benzel EC, Mroz TE (2015) Pseudoarthrosis rates in anterior cervical discectomy and fusion: a metaanalysis. Spine J 15:2016-2027

27. Hilibrand AS, Robbins M (2004) Adjacent segment degeneration and adjacent segment disease: the consequences of spinal fusion? Spine J 4:190S-194S

28. Shou F, Li Z, Wang H, Yan C, Liu Q, Xiao C (2015) Prevalence of $C 5$ nerve root palsy after cervical decompressive surgery: a meta-analysis. Eur Spine J 24:2724-2734

29. Clarke MJ, Ecker RD, Krauss WE, McClelland RL, DekutoskiMB (2007) Same-segment and adjacentsegment disease following posterior cervical foraminotomy. J Neurosurg Spine 6:5-9

30. Jödicke A, Daentzer D, Kästner S, Asamoto S, Böker DK (2003) Risk factors for outcome and complications of dorsal foraminotomy in cervical disc herniation. Surg Neurol 60:124-129 (discussion 129-30)

31. Kozak JA, Hanson GW, Rose JR, Trettin DM, Tullos HS (1989) Anterior discectomy, microscopic decompression, andfusion: a treatmentforcervical spondylotic radiculopathy. J Spinal Disord 2:43-46

32. Schöggl A, Reddy M, Saringer W, Ungersböck K (2002) Social and economic outcome after posterior microforaminotomy for cervical spondylotic radiculopathy. Wien Klin Wochenschr 114:200-204

33. Zhu Y, Zhang B, Liu H, Wu Y, Zhu Q (2016) Cervical disc arthroplasty versus anterior cervical discectomy and fusion for incidence of symptomatic adjacent segment disease: a meta-analysis of prospective randomized controlled trials. Spine (Phila Pa 1976) 41:1493-1502

\section{Notfallausweis fürs Pocket}

Klein, handlich und im entscheidenden Moment schnell griffbereit: Die Stadt Düsseldorf führt einen Notfallausweis im Pocketformat ein.

Der neue Düsseldorfer Notfallausweis ist knapp $11 \mathrm{~cm}$ hoch und knallgelb, passt in jedes Portemonnaie oder kann gut sichtbar und griffbereit ans Bett gelegt werden. So ist er für Rettungskräfte oder Angehörige im Notfall schnell einsichtig - anders als eine Patientenverfügung, die häufig erst gesucht werden muss und in einer Notfallsituation oft nicht beachtet wird.

\section{Patientenverfügung light}

Der Notfallausweis wird - wie auch die Patientenverfügung - zusammen mit dem Arzt und (wenn gewünscht) auch Angehörigen ausgefüllt und ist bindend. $\mathrm{Er}$ dient als Kurzzusammenfassung des Patientenwillens und gibt den Rettungskräften Rechtssicherheit. Damit soll noch besser dafür gesorgt werden, dass individuelle Wünsche berücksichtigt werden - quasi eine Patientenverfügung light.

\section{Besonders für Palliativpatienten}

Ausgestellt wird der neue Ausweis zunächst auf allen Düsseldorfer Palliativstationen sowie über das ambulant tätige Palliative Care Team. In einem nächsten Schritt ist dann die weitere Verbreitung über die niedergelassenen Allgemein- und Palliativmediziner im Stadtgebiet geplant. Entstanden ist der Ausweis in einer Kooperation der Stadt Düsseldorf und dem Runden Tisch Palliative Versorgung. $\mathrm{Zu}$ dem Zusammenschluss gehören neben den Krankenhäusern mit palliativer Versorgung der Landeshauptstadt u.a. auch Hospizverbände, Einrichtungen der Altenhilfe und die Krebsberatung Düsseldorf.

\section{Quelle: Universitätsklinikum Düsseldorf, www.uniklinik-duesseldorf.de}

